\title{
NOUVELLE
}

\section{Implication du processus de néogenèse lymphoïde dans le rejet chronique}

Olivier Thaunat, Anne-Christine Field, Jean-Baptiste Michel, Antonino Nicoletti

> La transplantation consiste à remplacer l'organe défectueux d'un patient par celui d'un autre individu. Cette idée, bien que très ancienne, n'est devenue une réalité clinique que depuis le milieu des années 1960. L'obstacle majeur auquel se sont heurtés les pionniers de la transplantation d'organe est le rejet. II résulte de la capacité du système immunitaire du receveur à discriminer comme étranger le tissu transplanté provenant d'un donneur de la même espèce génétiquement différent.

Tandis que les progrès constants dans le domaine de l'immunosuppression ont permis de contrôler efficacement le rejet aigu, la demi-vie des greffons ayant survécu à la première année a stagné, traduisant notre impuissance devant le processus de rejet chronique, devenu la première cause de perte des greffons. Notre incapacité à développer des stratégies thérapeutiques efficaces contre le rejet chronique est en bonne partie expliquée par notre ignorance de sa physiopathologie.

Sa définition reste en effet essentiellement histologique: survenue retardée d'une fibrose oblitérante progressive des structures canalaires du greffon, avec destruction du parenchyme et perte de fonction. La traduction clinico-histologique du rejet chronique dépend bien sûr de l'organe transplanté (rein, cœur, poumon...), cependant comme tous ces organes sont vascularisés, la composante vasculaire du rejet chronique (souvent appelée artériosclérose du greffon) est une constante. C'est ce caractère « universel » qui justifie que de très nombreuses équipes aient fait le choix de focaliser leurs travaux sur le rejet chronique vasculaire.

\section{Le rejet chronique vasculaire}

Un modèle largement utilisé pour étudier le rejet chronique vasculaire (RCV) consiste à réaliser une transplantation orthotopique de l'aorte abdominale sous-rénale entre deux souches de rat histo-incompatibles $(B N \rightarrow L \varepsilon W)$ [1]. Les lésions observées au niveau des greffons aortiques sont tout à fait superposables à celles observées au niveau des artères des organes détransplantés pour rejet chronique terminal chez l'homme. Ces lésions associent une prolifération intimale oblitérative, une disparition des cellules musculaires lisses de la média et une infiltration inflammatoire périvasculaire adventitielle.

Des travaux préliminaires nous ont appris que le développement des lésions de RCV, dans ce modèle, se déroulait en deux phases. La première se rapproche d'un rejet aigu : elle fait intervenir une réponse cellulaire cytotoxique responsable de la destruction rapide de l'endothélium du greffon. Elle est suivie par des processus de cicatrisation qui vont conduire à une prolifération néointimale oblitérative constituée par les CML (cellules musculaires lisses) du receveur (chimérisme). Les effecteurs cellulaires de cette première phase sont incapables d'atteindre les cibles allogéniques portées par les CML de la média qui sont protégées par les lames élastiques. Pour les détruire, le système immunitaire du receveur développe dans un deuxième temps une réponse humorale. Les alloanticorps traversent les lames élastiques, se fixent sur les molécules du complexe d'histocompatibilité de la classe I (CMH-I) allogéniques à la surface des CML médiales et induisent leur apoptose [2, 3]. L'importance de l'infiltrat inflammatoire adventitiel, disproportionné par rapport au faible nombre de cibles allogéniques présentes dans cette couche, et son caractère très chronologiquement corrélé aux évènements se déroulant dans les autres couches de la paroi artérielle, nous ont conduit à émettre l'hypothèse que cet infiltrat adventitiel pourrait jouer un rôle important dans le développement des lésions de rejet chronique vasculaire.

\section{Un processus de néogenèse lymphoïde}

Nous avons isolé l'adventice des greffons aortiques par microdissection, mis en suspension les cellules de l'infiltrat et analysé sa composition par cytométrie en flux [4]. Les lymphocytes $T$ constituent la population principale. Initialement (J10), l'infiltrat est majoritairement constitué de lymphocytes $T$ $\mathrm{CD}^{+}$et présente une polarisation marquée vers le phénotype Thl (T helper 1). Progressivement, la proportion de lymphocytes T $C D 4^{+}$augmente et une polarisation vers le phénotype Th2 (T helper 2) est observée à un mois. La population lymphocytaire $\mathrm{TCD}^{+}$semble fournir une aide au développement d'une réponse humorale alloimmune locale (néogenèse lymphoïde) [5]. En effet, nous avons observé, un mois après la transplantation, la présence dans l'adventice des greffons de nodules lymphocytaires $B$ (Figure IA) qui sont le siège d'une prolifération (FigurelB) et d'une apoptose intenses (Figure 1C), très évocatrices de centres germinatifs ectopiques. 
Le caractère fonctionnel de ces centres germinatifs adventitiels a été documenté par des techniques d'organoculture en condition hyperoxique qui ont permis de démontrer qu'ils sont le siège de la production d'alloanticorps.

Enfin, nous avons mis en évidence un tissu lymphoïde secondaire ectopique au sein de greffons humains (reins et cœurs) détransplantés pour rejet chronique terminal, démontrant la réalité $d u$ processus de néogenèse lymphoïde en transplantation clinique.

Ces résultats démontrent que les greffons sont non seulement la cible mais aussi le site où la réponse allo-immune

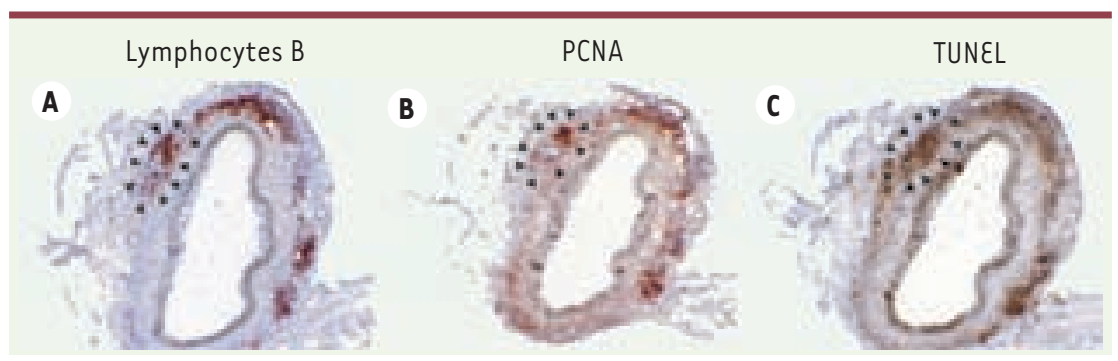

Figure 1. Coupe transversale de greffon aortique un mois après la transplantation. PCNA : proliferating cell nuclear antigen; TUNEL : terminal deoxynucleotidyl transferase-mediated dUTP nick end labelling.

s'élabore. De futurs travaux devront vérifier si la néogenèse lymphoïde est une cible thérapeutique intéressante pour prévenir le rejet chronique. $\diamond$ Implication of the lymphoid neogenesis process in chronic rejection

\section{RÉFÉRENCES}

1. Mennander A, Tiisala S, Halttunen J, et al. Chronic rejection in rat aortic allografts. An experimental model for transplant arteriosclerosis. Arterioscler Thromb 1991; 11: 671-80.

2. Plissonnier D, Henaff M, Poncet $P$, et al. Involvement of antibody-dependent apoptosis in graft rejection. Transplantation $2000 ; 69: 2601-8$.

3. Plissonnier D, Nochy D, Poncet $P$, et al. Sequential immunological targeting of chronic experimental arterial allograft. Transplantation 1995 ; 60 : 414-24.

4. Thaunat 0, Field AC, Dai J, et al. Lymphoid neogenesis in chronic rejection : evidence for a local humoral alloimmune response. Proc Natl Acad Sci USA 2005; 102 : 14723-8.

5. Kratz A, Campos-Neto A, Hanson MS, et al. Chronic inflammation caused by lymphotoxin is lymphoid neogenesis. J Exp Med 1996; 183 : 1461-72.

\title{
NOUVELLE
}

\section{Les bactériophages de type T4 Des composants prépondérants de la 《matière noire » de la biosphère}

Jonathan Filée, André M. Comeau, Curtis A. Suttle, Henry M. Krisch

> La biosphère a aussi sa masse cachée: les bactériophages. Nombreux, omniprésents et inertes, les bactériophages ne deviennent actifs que lorsqu'ils infectent une bactérie hôte parmi les innombrables bactéries présentes dans l'environnement. Les phages sont les entités les plus abondantes de la biosphère: on estime que leur nombre dépasse celui des bactéries d'un facteur 10 environ [1, 2]. Plus de 5000 phages ont été décrits dans la littérature, $96 \%$ d'entre eux possèdent une queue [3]. Mais cet inventaire ne représente qu'une fraction infime de la diversité réelle des phages. II existerait plus de $10^{30}$ phages à queue dans la biosphère [4]. Parmi ceux-ci, les myovirus à queue contractile sont particulièrement répandus. Des études quantitatives par

microscopie électronique et

\author{
J. Filée, A.M. Comeau, H.M. Krisch : \\ Laboratoire de Microbiologie et de Génétique moléculaire, \\ CNRS, UMR5100, 118, route de Narbonne, \\ 31062 Toulouse Cedex 9, France. \\ C.A. Suttle : Department of Earth \\ and Ocean Sciences (Oceanography), \\ University of British Columbia, \\ 6270 University Boulevard, Vancouver BC, V6T 1 Z4 Canada. \\ jonathan.filee@ibcg.biotoul.fr
}

des analyses métagénomi-

ques indiquent que, dans les environnements marins, les myovirus représentent une fraction prépondérante des phages présents.

T4, l'archétype de la famille des myovirus, appartient au groupe monophylétique des phages de type T4 [5]. Sur la base de leur séquence et de leur morphologie, les phages de type T4 peuvent être classés en différents sous-groupes ( $T$ pairs et pseudo-T pairs, schizo et exo- $T$ pairs). L'analyse récente de séquences $d u$ génome entier d'une douzaine de phages de type T4 a largement accru nos connaissances de la superfamille T4. Mais l'estimation de la diversité des phages dans la nature reste toujours mal connue car beaucoup de bactéries de l'environnement (et de ce fait leurs phages) ne sont pas cultivables. Pour contourner cette difficulté, des analyses de séquences génétiques ont été faite directement sur de l'ADN extrait de l'environnement et amplifié sélectivement par PCR (polymerase chain reaction), sans passer par l'étape de mise en culture.

Étonnamment, la partie centrale de la séquence du gène codant pour la protéine principale (g23) de la tête du phage T4 (la capside) peut servir de bon substitut phylogénique à l'ensemble du génome [6]. Nous avons donc créé une paire 